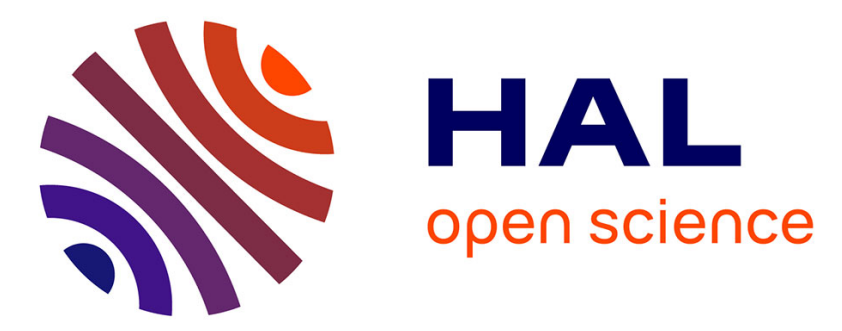

\title{
Experimental validation of an info-gap uncertainty model for a robustness analysis of structural responses
}

Antoine Kuczkowiak, Scott Cogan, Morvan Ouisse, Emmanuel Foltête, Mathieu Corus

\section{- To cite this version:}

Antoine Kuczkowiak, Scott Cogan, Morvan Ouisse, Emmanuel Foltête, Mathieu Corus. Experimental validation of an info-gap uncertainty model for a robustness analysis of structural responses. Journal of Risk and Uncertainty in Engineering Systems, Part B: Mechanical Engineering, 2020, 6, pp.11. hal-02993927

\section{HAL Id: hal-02993927 \\ https://hal.science/hal-02993927}

Submitted on 7 Nov 2020

HAL is a multi-disciplinary open access archive for the deposit and dissemination of scientific research documents, whether they are published or not. The documents may come from teaching and research institutions in France or abroad, or from public or private research centers.
L'archive ouverte pluridisciplinaire HAL, est destinée au dépôt et à la diffusion de documents scientifiques de niveau recherche, publiés ou non, émanant des établissements d'enseignement et de recherche français ou étrangers, des laboratoires publics ou privés. 


\title{
Experimental validation of an info-gap uncertainty model for a robustness analysis of structural responses
}

\author{
Antoine Kuczkowiak \\ Scott Cogan \\ Dr. Ing. \\ CNRS Research fellow \\ FEMTO-ST \& EDF LAB Paris-Saclay Univ. Bourgogne Franche-Comté \\ IMSIA - UMR EDF-CNRS-CEA-ENSTA \\ FEMTO-ST Institute \\ ParisTech 9219 \\ CNRS/UFC/ENSMM/UTBM \\ Universit Paris-Saclay \\ 7 boulevard Gaspard Monge \\ 91120 Palaiseau, France \\ antoine.kuczkowiak@edf.fr \\ Department of Applied Mechanics \\ 24 rue de l'Épitaphe \\ 25000 Besançon, France \\ scott.cogan@univ-fcomte.fr

\section{Morvan Ouisse} \\ Professor \\ Univ. Bourgogne Franche-Comté \\ FEMTO-ST Institute \\ CNRS/UFC/ENSMM/UTBM \\ Department of Applied Mechanics \\ 24 rue de l'Épitaphe \\ 25000 Besançon, France \\ morvan.ouisse@femto-st.fr

\section{Emmanuel Foltête} \\ Professor \\ Univ. Bourgogne Franche-Comté \\ FEMTO-ST Institute \\ CNRS/UFC/ENSMM/UTBM \\ Department of Applied Mechanics \\ 24 rue de l'Épitaphe \\ 25000 Besançon, France \\ emmanuel.foltete@femto-st.fr
}

\section{Mathieu Corus}

Dr. Ing.

EDF LAB PARIS-SACLAY

Boulevard Gaspard Monge

91120 Palaiseau, France

mathieu.corus@edf.fr

\section{ABSTRACT}

The aim of this work is to propose an indicator, based on the info-gap approach, which 
assesses the robustness of the dynamic response of the model of a structure to lack of knowledge in the associated eigensolutions. The info-gap uncertainty model for the eigensolutions is constructed mode by mode based on a set of experimentally identified eigensolutions obtained from tests on a set of nominally identical structures. A robustness anal$y$ sis is then performed which provides a useful bound to the maximum response levels that are consistent with the defined uncertainty, thus allowing to extrapolate to a population of untested structures. The proposed methodology is validated experimentally on a simple structure composed of two plates clamped together on one side. Uncertainty is introduced by adding lumped masses at random locations. A subset of these test results is used to construct the info-gap model of the eigenproperties and the remaining data is used to confirm that the robustness curve usefully bounds the observed maximum responses.

\section{INTRODUCTION}

Structural dynamic behaviors must be well understood in order to ensure the reliable and safe operation of complex systems. When available, validated numerical models are useful for predicting vibratory levels under both tested and untested conditions. However, the complexity and the degree of epistemic uncertainty, due to the difficulty in modeling poorly known physics, can render the model validation process excessively time consuming and costly. Moreover, experimental modal analyses are often performed in a configuration which differ significantly from real operating conditions. For example, the configuration of GVT (Ground Vibration Testing) used to identify normal modes on a aircraft differs from the conditions encountered during flight. Another example is an alternator in a power plant where experimental modal analyses are performed on a non-operating machine while it is known that thermal and nonlinear mechanical effects of the functioning alternator modify the dynamic behavior. While in the case of the aircraft testing, operational modal analysis provides a way to obtain the eigensolutions during flight, in the case of the alternator, this is not possible.

In this paper, the identified modal model is considered to be uncertain and is assumed to be a 
direct result of the lack of knowledge between two configurations. Broadly speaking, the present study is directly related to the field of robustness analysis which aims is to provide decision support tools in order to support decision under deep uncertainty. The notion of robustness can take on many meanings as discussed in the papers by Göhler et al [23] and Zhang et al [24]. In the context of this work, a non-probabilistic notion of robustness will be employed as defined in the framework of the info-gap theory [1]. Knight [2] distinguished between "risk" based on known probability distributions and "true uncertainty" for which probability distributions are not known. In the present work, robustness is defined as an aptitude to resist to approximations or zones of ignorance in order to prevent undesirable impacts [3]. Robustness analyses are performed within the framework of model predictions corresponding to a posteriori actions. Reliable robustness analyses can be performed only when the uncertainty in the system can be represented as a mathematical idealization. A well-established classification of uncertainty permits to make a clear distinction between aleatory and epistemic uncertainty [4-6]. Aleatory uncertainty is also referred to as irreducible uncertainty since it always exists by nature and can not be suppressed even when more accurate experiments are performed. The mathematical idealization of such uncertainties is often a probability or frequency distribution [7-10]. Epistemic uncertainty deals with lack of knowledge about the system and/or the environment and is also referred to as reducible uncertainty since more accurate experiments could reduce them. A wide variety of approaches have been developed to treat epistemic uncertainty, including interval [11], fuzzy [12], Dempster-Shafer [13,14] or info-gap theory [1]. The industrial issue of interest in this study concerns a modal model of a turboalternator. This model is assumed to be uncertain as it is obtained from an experimental modal analysis under non-operating conditions. Moreover, no model is currently available to predict the evolution of the modal basis when the alternator is in operation. In this context, the uncertainty in the identified eigensolutions are considered to be epistemic. In this paper, an info-gap robustness framework will be adopted to investigate the impact of this epistemic uncertainty on the response levels of interest. As with any uncertainty propagation technique, the results depends strongly on the info-gap model of uncertainty used to represent the lack of knowledge affecting the eigensolutions. The objective of this paper is to validate experimentally, on the basis of an academic test 
case, the model of uncertainty selected for the robustness analysis. Very few studies are currently available in the literature that investigate this question for the info-gap approach. [15] or [16, 17] address an analogous problem of validation of a non-parametric model of uncertainty based on real measurements.

The paper is organized as follows. Section 2 introduces the methodology to assess the robustness of the dynamic response levels to uncertain eigensolutions. Section 3 proposes a numerical illustration. Finally, section 4 proposes a way to construct and calibrate a model of uncertainty based on a subset of test-data. Finally, the selected uncertainty model is validated using on real experiments.

\section{ROBUSTNESS OF DYNAMIC RESPONSES TO UNCERTAIN EIGENSOLUTIONS}

The approach adopted here consists in synthesizing the dynamic response of a linear elastodynamic structure based on uncertain eigensolutions. The impact of lack on knowledge in the eigenparameters is then investigated using an info-gap robustness analysis [1]. The info-gap methodology is a decision theory and aims to analyze the robustness of a decision under severe uncertainties. It has been applied in very diverse domains including economics, medicine and business management as its formulation is very general. In the field of structural dynamics, a wide variety of applications can be found in the literature, for example [25, 26, 28, 28], and readers can refer to one of these references for a more thorough introduction to the info-gap methodology if required.

The info-gap robustness analysis requires the definition of three essential components, namely, the system model, the info-gap model of uncertainty and the performance requirements. The robustness function is based on the concept of "satisficing" ${ }^{1}$ performance requirements, that is to say, insuring a given level of performance even under a given level of uncertainty.

\footnotetext{
${ }^{1}$ Satisficing is the contraction of satisfactory and sufficient.
} 


\subsection{System model}

\subsection{System model}

The system model establishes the functional relationship between the uncertain model parameters, $\boldsymbol{x}$, the known model parameters, $\boldsymbol{p}$, and the response features of interest, $\boldsymbol{y}$. It is written as

$$
\mathcal{M}(\boldsymbol{p}, \boldsymbol{x})=\boldsymbol{y}
$$

In many structural applications, $\mathcal{M}$ represents a physics-based model of a structure, $\boldsymbol{x}$ the model input parameters (eg geometric dimensions, plate thicknesses or material properties) and $\boldsymbol{y}$ the model outputs or structural responses of interest (eg frequency responses, eigenfrequencies or stresses). But the concept of a system model is very general and can be seen simply as a blackbox representation relating inputs and outputs via a more or less complex functional relationship $\mathcal{M}$. In the present case, $\mathcal{M}$ will represent the relationship between the eigenparameters $x$ of a mechanical structure and its frequency responses $y$.

In the low frequency domain, the modal behavior controls the mechanical response of a given system. Experimental modal analyses are used to obtain the spectrum of the structure. Let $\left(\omega_{\nu}^{0}, \xi_{\nu}^{0}, \phi_{\nu}^{0}\right) \in \mathbb{R} \times \mathbb{R} \times \mathbb{R}^{c}$ be an experimentally identified modal basis with $\omega_{\nu}^{0}$ the eigenfrequency, $\xi_{\nu}^{0}$ the modal damping factor, $\phi_{\nu}^{0}$ the eigenvector corresponding to the mode $\nu(\nu \in\{1, \cdots, m\})$ and $c$ the number of sensors. The incomplete identified modal basis can be used to evaluate the dynamic response levels $\boldsymbol{y}$ at the frequency $\omega$ subjected to a dynamic load $\boldsymbol{F} \in \mathbb{R}^{c}$ at the sensors degrees of freedom (dofs):

$$
\boldsymbol{y}=\left|\sum_{\nu=1}^{m} \frac{\phi_{\nu}^{0}\left(\phi_{\nu}^{0}\right)^{T} \boldsymbol{F}}{-\omega^{2}+2 \mathrm{\jmath} \xi_{\nu}^{0} \omega \omega_{\nu}^{0}+\left(\omega_{\nu}^{0}\right)^{2}}\right|
$$

In what follows, equation (2) is the system model. 


\subsection{Info-gap model of uncertainty}

\subsection{Info-gap model of uncertainty}

The info-gap model of uncertainty defines an unbounded family of nested sets of realizable designs. The size of these sets is controlled by the horizon of uncertainty and denoted $\alpha(\alpha \in \mathbb{R}+)$. The horizon of uncertainty indicates the level of uncertainty present in the model of uncertainty. A wide variety of info-gap uncertainty models are available in the literature [1]. The choice of the model of uncertainty must be performed with care since it is known that the info-gap robustness analysis is sensitive to the model of uncertainty. In this paper, the identified modal basis is assumed to be uncertain. Let $\left(\omega_{\nu}, \xi_{\nu}, \phi_{\nu}\right) \in \mathbb{R} \times \mathbb{R} \times \mathbb{R}^{c}$ be the uncertain modal basis. The uncertainty model is derived from the nominal modal basis by assuming that the uncertainty is driven by the vector $\boldsymbol{x} \in \mathbb{R}^{p}$ with $p=2 m+m^{2}$. This column vector is defined by

$$
\mid \begin{aligned}
& \omega_{\nu}=x_{\nu} \omega_{\nu}^{0}, \quad \forall \nu \in\{1, \cdots, m\} \\
& \xi_{\nu}=x_{\nu+m} \xi_{\nu}^{0}, \quad \forall \nu \in\{1, \cdots, m\} \\
& \phi_{\nu}=\sum_{k=1}^{m} x_{(1+\nu) m+k} \phi_{k}^{0}, \quad \forall \nu \in\{1, \cdots, m\}
\end{aligned}
$$

where $x_{\nu}$ is the $\nu$-th component of $\boldsymbol{x}$. The uncertain eigenvector is assumed to be described by the initial eigenvectors (assumption valid for small uncertainties and decoupled pairs of eigenvectors [18]). In addition, the particular column vector which corresponds to a zero-perturbation applied on the modal basis is denoted $x^{0} \in \mathbb{R}^{p}$ and is defined in such way to recover the initial modal basis $\left(\omega_{\nu}^{0}, \phi_{\nu}^{0}, \xi_{\nu}^{0}\right)$ with $\nu \in\{1, \cdots, m\}$. This vector is not assumed to be uncertain contrary to the vector $\boldsymbol{x}$. For example, in the case where $m=3$ modes are taking into account in the analysis, the initial vector $\boldsymbol{x}^{0} \in \mathbb{R}^{p}$, with $p=15$ and is defined by

$$
\boldsymbol{x}^{0}=(\underbrace{1,1,1}_{\text {Perturbation on } \omega_{\nu}^{0}, \nu \in\{1,2,3\}}, \overbrace{1,1,1}^{\text {Perturbation on } \xi_{\nu}^{0}, \nu \in\{1,2,3\}}, \underbrace{1,0,0,0,1,0,0,0,1}_{\text {Perturbation on } \boldsymbol{\Phi}^{0}=\left[\boldsymbol{\phi}_{1}^{0} \boldsymbol{\phi}_{2}^{0} \boldsymbol{\phi}_{3}^{0}\right]})^{T} .
$$




\subsection{Performance requirement}

The lack of knowledge in the eigenparameters is represented by an absolute error model:

$$
\mathcal{U}\left(\alpha, \boldsymbol{x}^{0}\right)=\left\{\boldsymbol{x} \in \mathbb{R}^{p}:\left|\boldsymbol{x}-\boldsymbol{x}^{0}\right| \leq \alpha \boldsymbol{w}\right\}
$$

where

$$
\mid \begin{aligned}
& \alpha \geq 0, \text { the horizon of uncertainty; } \\
& \boldsymbol{w} \geq 0, \text { a vector of weighting coefficients }\left(\boldsymbol{w} \in \mathbb{R}^{p}\right) .
\end{aligned}
$$

In other words, the greater the horizon of uncertainty $\alpha$, the greater the Euclidean distance between the vectors $x$ and $x^{0}$ can be. The vector of weighting coefficients, which defines the uncertainty model (equation (5)), expresses the relative confidence between the different eigenparameters. It can be assessed in different ways: on the basis of engineering judgment when information is lacking or by using the empirical robustness. This point will be discussed in section 4.3.

\subsection{Performance requirement}

The performance requirements represent the quantity of interest upon which a decision or set of decisions will be based. The performance requirement is sometimes called the decision-making model since the decision is based on the specification of a critical level. In the present case, the maximum response level of the system $y$ at one frequency of interest $\omega_{0}$ (or possibly a frequency band of interest) must be less than or equal to some critical value $y^{c}$

$$
y \leq y^{c}
$$

In practice, $y$ is built from the components of $y$ defined by the equation (2). In what follows, the equation (6) is the decision-making model. 


\subsection{Info-gap robustness}

\subsection{Info-gap robustness}

"Uncertainty may be either pernicious or propitious" (p. 37 in [1]). While this may seem like a trivial observation, info-gap decision theory addresses the problem of quantifying these two aspects of uncertainty by two "immunity functions": the robustness function, which expresses the immunity to failure, and the opportuneness function, which expresses the immunity to windfall gain. The present study only deals with robustness function. The robustness function expresses the greatest horizon (level) of uncertainty $\hat{\alpha}$ that can be tolerated without exceeding the critical performance requirement $y^{c}$. Thus, $\hat{\alpha}$ depends intimately on $y^{c}$ and is formally defined by [1]

$$
\hat{\alpha}\left(y^{c}\right)=\underset{\alpha \geq 0}{\arg \max }\left\{\max _{\boldsymbol{x} \in \mathcal{U}\left(\alpha, \boldsymbol{x}^{0}\right)} y \leq y^{c}\right\} .
$$

However, for a given performance requirement, the costly non linear optimization problem (7) must be solved in order to obtain a value of robustness. While an analytical expression of the robustness function may be available in simple cases where the structural dynamic behavior is controlled by few well-separate modes, a more general approach is employed here. Practically speaking, rather than fix $y^{c}$, it is easier to fix $\hat{\alpha}$. The robustness is thus discretized by a series of increasing values:

$$
\hat{\alpha}=\left\{0, \cdots, \hat{\alpha}_{i}, \cdots\right\}
$$

and, for each $\hat{\alpha}_{i}$ an optimization problem under constraints is solved:

$$
y^{c}\left(\hat{\alpha}_{i}\right)=\max _{\boldsymbol{x} \in \mathcal{U}\left(\hat{\alpha}_{i}, \boldsymbol{x}^{\mathbf{0}}\right)} y
$$

The solution is obtained by using the interior-point method which is a local optimization algorithm from the fmincon function available in the Matlab ${ }^{\circledR}$ Optimization ToolBox. 
The info-gap decision theory is not directly related to uncertainty quantification/propagation. The main interest of such an approach is to study the different trade-offs involved in making decision under lack of knowledge. One of the most important trade-off is that the robustness and the performance requirement are antagonistic: if small values of $y^{c}$ are required then only low robustness (immunity) to uncertainty is possible. The simplest way to explore this trade-off is obtained by the robustness curve, which is the graphical representation of $\hat{\alpha}=f\left(y^{c}\right)$. Nonetheless, it should be emphasized that solving equation (9) is equivalent to solving equation (7).

\section{NUMERICAL ILLUSTRATION}

In this section, an illustration, based on finite element (FE) model predictions, is proposed. The system is composed of two $700 \times 440 \times 5 \mathrm{~mm}^{3}$ aluminum plates clamped together on one edge and at three stacking points (cf. Figure 6). The system is assumed to be in free-free configuration. The FE model (cf. Figure 1) is composed of approximately 3000 nodes. A numerical modal basis is thus obtained and a robustness analysis is performed in order to assess the maximum level of uncertainty on the modal basis that the system can tolerate without exceeding a critical response level. For the sake of clarity, only the first eigenfrequency is assumed to be uncertain. As shown

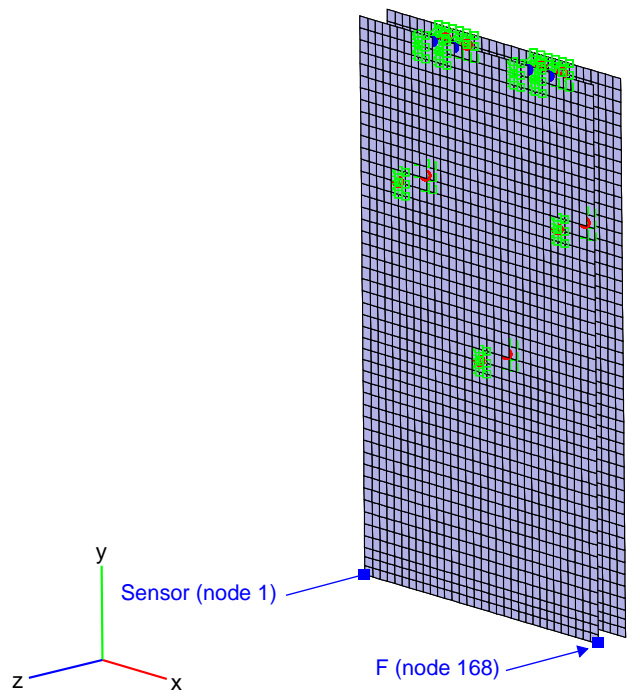

Fig. 1. FE model

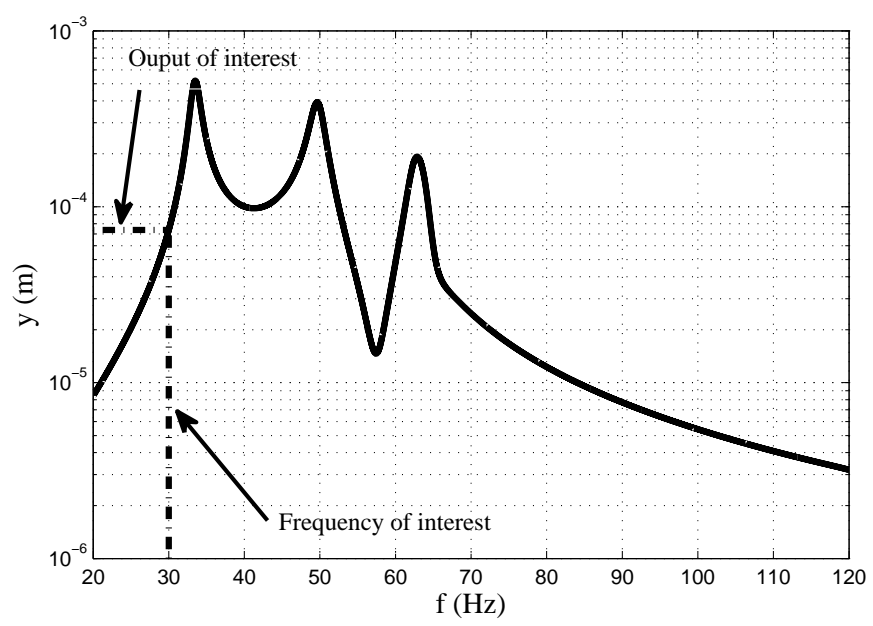

Fig. 2. FRF between the node 1 (direction $z$ ) and the node 168 (direction $z$ ) 
in Figure 1, a transverse unit point force is located on node 168 (direction z) while the transverse response displacement amplitude is observed at the node 1 (direction $\mathrm{z}$ ). The modal basis used to assess the response of the system is composed of five modes and is depicted in Figure 3 . The Figure 2 plots the FRF between the input/output of the system. In the present study, the output of interest is the response displacement amplitude at the frequency of interest $f=30 \mathrm{~Hz}$.

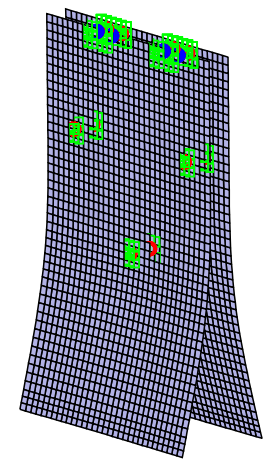

Mode 1: $33 \mathrm{~Hz}$

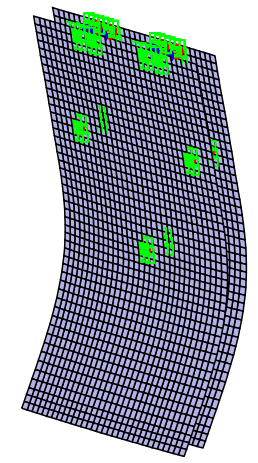

Mode 4: $64 \mathrm{~Hz}$

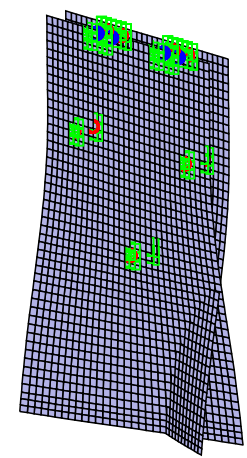

Mode 2: $50 \mathrm{~Hz}$

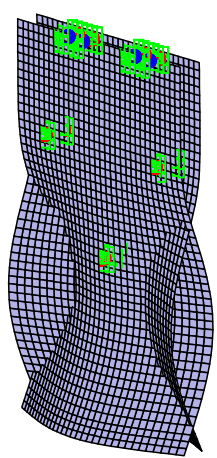

Mode 5: $119 \mathrm{~Hz}$

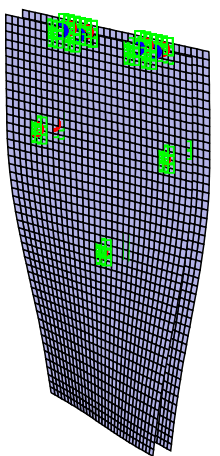

Mode 3: $62 \mathrm{~Hz}$

Fig. 3. Mode shapes used to assess the robustness of the system

The robustness curve is the black curve in Figure 4 and is obtained for an horizon of uncertainty from 0 to 0.2 with a step $\alpha_{i}=0.001$. This curve is evaluated point by point using optimization methods. At a given horizon of uncertainty $\alpha_{i}$ :

1. The input parameter in the optimization problem is the eigenfrequency of the first elastic mode 
that can vary between $\left(1-\alpha_{i}\right) f_{1}^{0} \leq f_{1} \leq\left(1+\alpha_{i}\right) f_{1}^{0}$, where $f_{1}^{0}$ is the value of the frequency for the nominal model.

2. The objective function to be maximized is the displacement amplitude at the node 1 along the $z$-direction

3. The box constraints on the realizable eigenfrequency value depend directly on the current horizon of uncertainty

4. A local nonlinear optimization algorithm is used to maximize the objective function thus providing the largest displacement at $\alpha_{i}$.

5. $\alpha_{i}$ is incremented a step and the next optimization problem is solved over the new domain.

In the present case, the robustness curve is composed of 2 zones:

$\hat{\alpha} \in\left[0 ; \hat{\alpha}_{1}\right]$ : the displacement amplitude increases significantly with increasing horizons of uncertainty. This is evidently due to the fact that the peak response at the resonance is moving closer and closer to the frequency of interest situated at $30 \mathrm{~Hz}$. The performance in this zone is clearly vulnerable to increasing uncertainty.

$\hat{\alpha} \in\left[\hat{\alpha}_{1} ; 0.2\right]:$ the maximal displacement amplitude does not change since the horizon of uncertainty is large enough for the first eigenfrequency to shift to $f=30 \mathrm{~Hz}$. Larger horizons of uncertainty in this simple test case will not lead to a worse case. This explains why the robustness curve is vertical for $\hat{\alpha} \geq \hat{\alpha}_{1}$ meaning that increasing horizon of uncertainty no longer has an impact on the worst case maximum response. As such, the performance in this zone is seen to be robust to uncertainty.

In this simple case, it is can be concluded that if a response amplitude of $y_{1}$ is acceptable than it is shown to be robust to the uncertainty in the first eigenfrequency. Here the robustness curve obtained by optimization methods can be validated using a more basic approach. Since there is only one uncertain eigenparameter, namely, the first eigenfrequency, we can model this uncertainty as an interval with the worst case found at each boundary of the interval. The simulated validation data using this approach, indicated by the red points in Figure 4, yields the same maximum response levels at $f=30 \mathrm{~Hz}$ when the first eigenfrequency $f_{1}$ is in the interval 


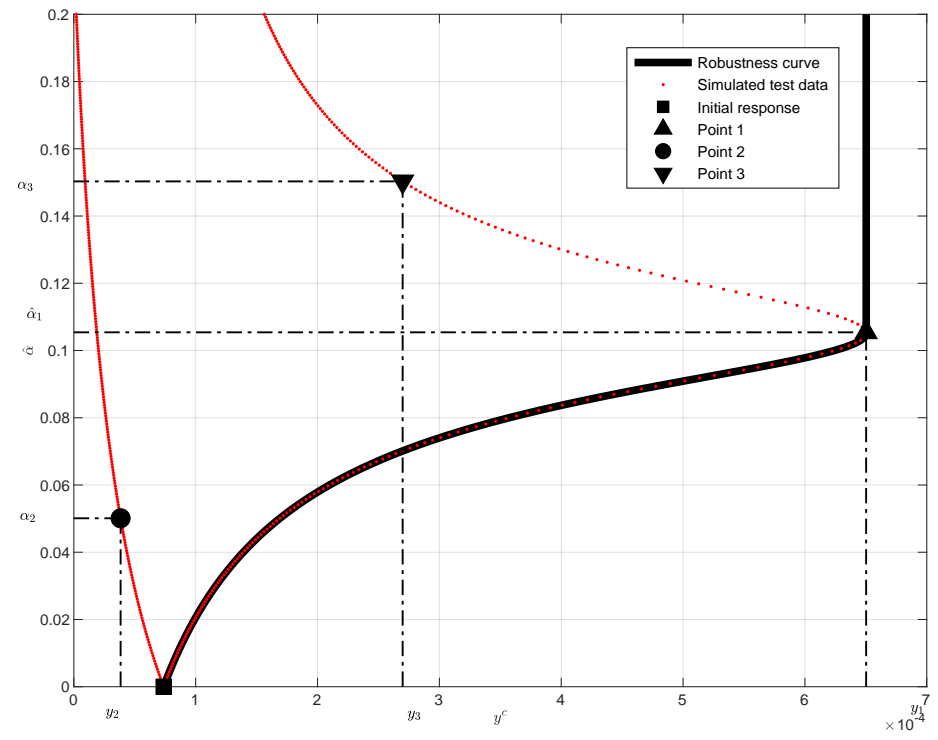

Fig. 4. Robustness curve

$\left[f_{1}(1-\hat{\alpha}) ; f_{1}(1+\hat{\alpha})\right]$. In this case, the robustness curve matches the simulated validation data in $\left[0 ; \hat{\alpha}_{1}\right]$ plotted as the black curve. The opportuness curve ( $\alpha_{2}$ on the red points), represents the best-case lowest amplitude response level for inceasing horizon of uncertainty. However, the most defavorable response levels are not necessarily at the boundary of the uncertainty domain. This can be seen in the upper red curve for $\alpha_{3}$ which represents the worst case at the boundary 
of the interval. It is evident here that the worst case is still found for $\hat{\alpha}=\hat{\alpha}_{1}$, as indicated in Figure 5 .

The initial response corresponds to the amplitude of the response at the frequency $f=30 \mathrm{~Hz}$ when no uncertainty is taken into account $(\alpha=0)$. To summarize, when the horizon of uncertainty increases there are two possibilities. The point 2 corresponds to the best possibility since the amplitude at $f=30 \mathrm{~Hz}$ is lower than the original one. The point 1 is the worst possibility when $\alpha=\alpha_{1}$ since the amplitude at $f=30$ is larger than the original one.

This simple numerical example illustrates the approach that will be adopted in this study. However in real life applications the uncertainty in a set of eigenparameters must be taken into account, including eigenfrequencies, eigenvectors and modal damping factors.

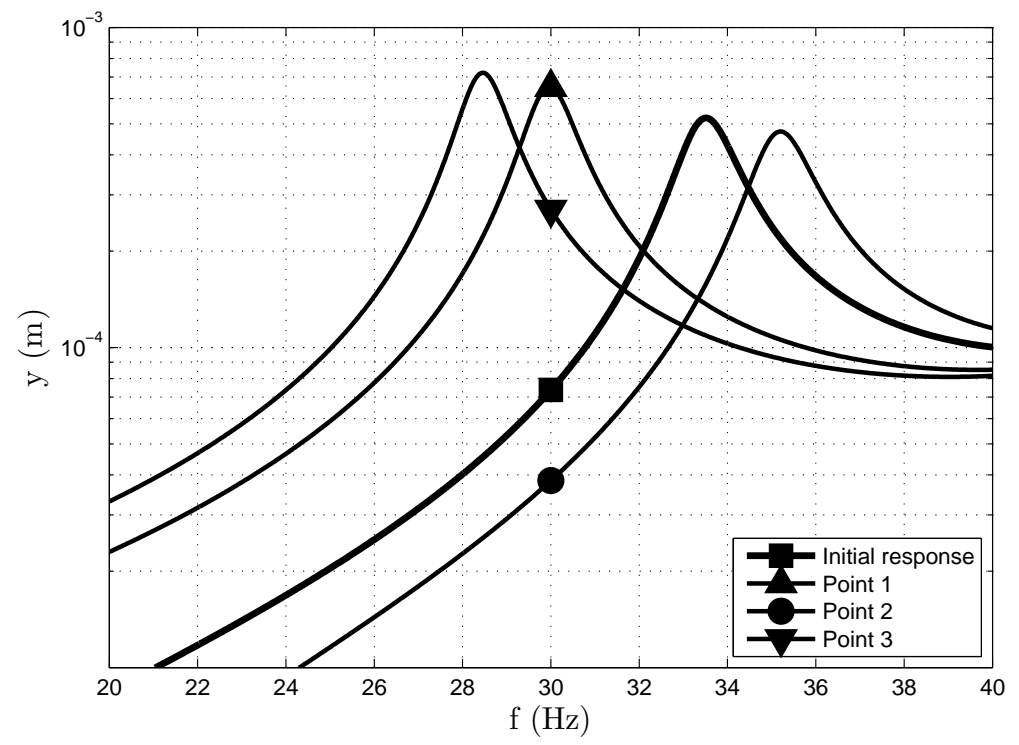

Fig. 5. Real FRFs for checking the accuracy of the robustness curve

\section{EXPERIMENTAL VALIDATION}

As with stochastic approaches, the results of an info-gap robustness analysis are sensitive to the underlying uncertainty model used in the analysis. The objective of this section is to detail the 
construction of an info-gap model of uncertainty based on a subset of test data. As an illustration, the methodology is applied to the experimental structure shown in Figure 6, which is the structure described in section 3. The system is instrumented with five accelerometers on the free edges of each plate and with an impedance head at the excitation point. An experimental modal analysis is carried out using a low level white noise excitation from 5 to $312 \mathrm{~Hz}$. For the purposes of validation, 32 experimental modal analyses are performed, each one corresponding to a different configuration. The configurations differ from each other due to local perturbations created with lumped masses introduced in random locations to modify the eigenparameters of the baseline structure and the resulting modal bases have been identified. Section 4.1 demonstrates that the uncertainties in the system are only the consequences of the addition of lumped masses in the system. Section 4.2 aims to express the variability in the modal basis when the whole set of configurations are considered. Furthermore, this section demonstrates that the uncertainty resulting from added masses does not have the same impact on the variability of the different eigenparameters. Section 4.3 describes the construction and calibration of the info-gap model of uncertainty in order to validate it in section 4.4 .

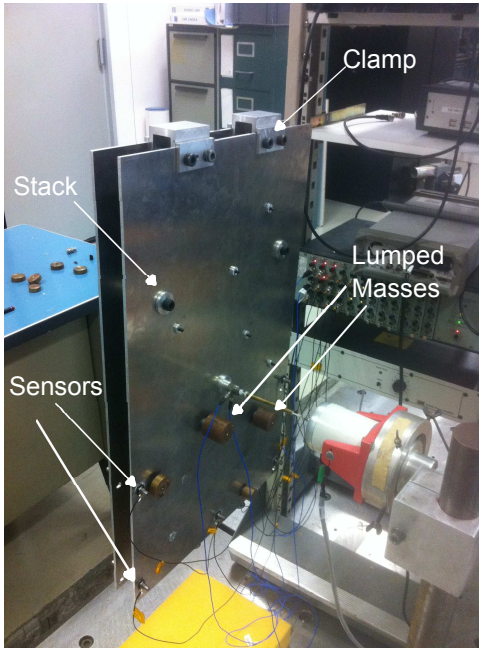

Fig. 6. Experimental set-up

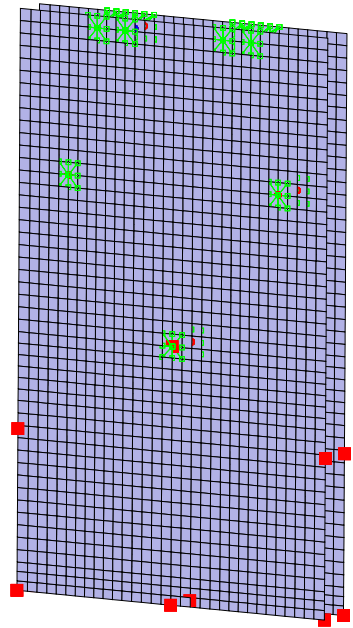

Fig. 7. Location of the sensors (red square) projected onto the FE model (dir z) 


\subsection{Modal Identification}

\subsection{Modal Identification}

The objective of this section is to demonstrate that the dominant uncertainties in the system under study are only the consequences of the addition of lumped masses in the system. Hence, the modal identification should be as rigorous as possible in order to minimize the errors during the identification process. In the present study, the modal identification is performed in two steps:

1. Pole/residue model identification by using LSCF (Least Square Complex Frequency) method [19]. The measured Frequency Response Functions are synthetized by

$$
\mathrm{FRF}_{i j}^{i d}=\sum_{\nu=1}^{N} \frac{R_{\nu}^{i j}}{s-\lambda_{\nu}}+\frac{R_{\nu}^{i j *}}{s-\lambda_{\nu}^{*}} \in \mathbb{C}
$$

with

$s=\mathrm{j} \omega:$ the Laplace variable;

$N$ : the number of identified poles;

$\lambda_{\nu}=-\xi_{\nu} \omega_{\nu}+j \omega_{\nu} \sqrt{1-\xi_{\nu}^{2}} \in \mathbb{C}$ : the $\nu$-th identified pole ${ }^{2}$

$R_{\nu}^{i j} \in \mathbb{C}$ : the identified residue corresponding to the mode $\nu$ between the input $i$ and the output $j$. The complex mode $\psi_{\nu}$ is derived from the residue matrix $\boldsymbol{R}_{\nu}$ by $\boldsymbol{R}_{\nu}=\boldsymbol{\psi}_{\nu}\left(\boldsymbol{\psi}_{\nu}^{*} \boldsymbol{F}\right)$ where $\left(\boldsymbol{\psi}_{\nu}^{*} \boldsymbol{F}\right)$ is the modal factor ${ }^{3}$.

2. Normal mode approximations derived from the complex modes where the synthesis is rewritten using the normal eigenvectors $\phi_{\nu}$ :

$$
\mathrm{FRF}_{i j}^{n m}=\sum_{\nu=1}^{N} \frac{\phi_{\nu}^{i}\left(\phi_{\nu}^{j}\right)^{T}}{s^{2}+2 \xi_{\nu} \omega_{\nu} s+\omega_{\nu}} \in \mathbb{C}
$$

As shown in Figure 8, the measured FRFs have an acceptable signal/noise ratio. Furthermore, due to the simplicity of the tested structure, the mode complexity is very low. Figure 9 plots

\footnotetext{
${ }^{2}$ The modal damping assumption is supposed to be valid.

${ }^{3}$ Here, $\left(\boldsymbol{\psi}_{\nu}^{*} \boldsymbol{F}\right) \in \mathbb{R}$ since a mono-excitation (punctual force) is applied on the structure.
} 


\subsection{Modal Identification}

the complex vectors in the Nyquist map and almost no phase difference between the sensors is found. After pole/residue model identification, complex mode are normalized with regards to the

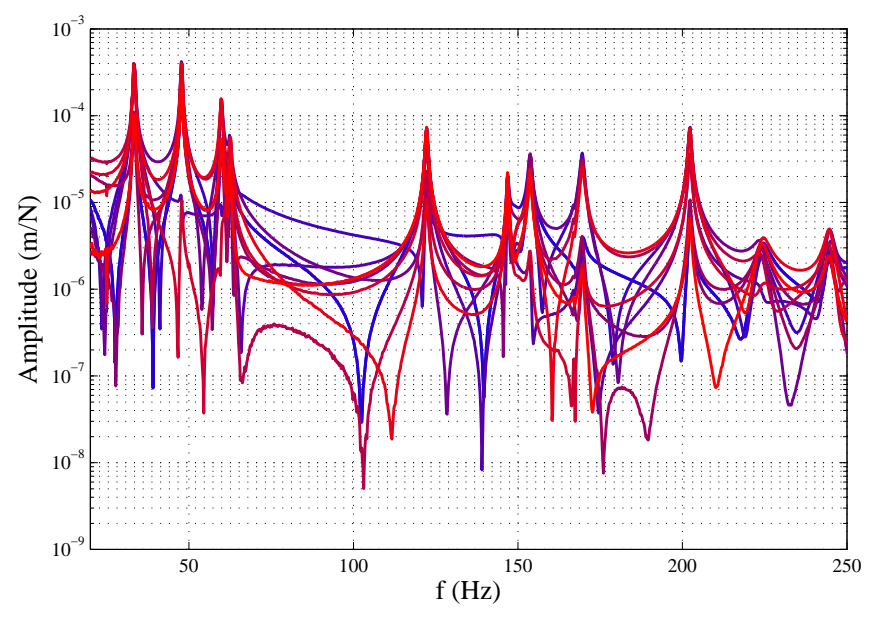

Fig. 8. Measured FRFs

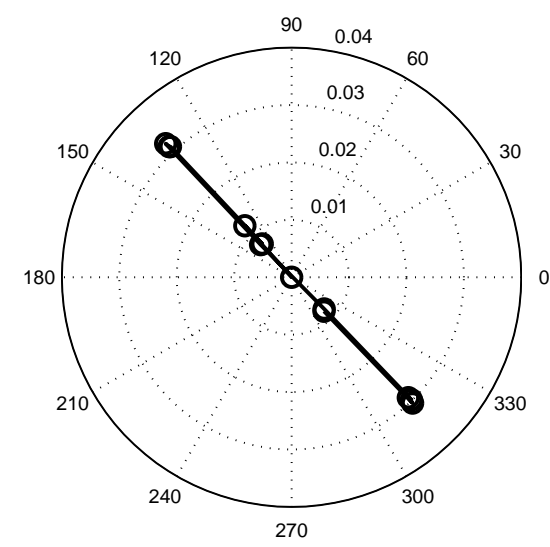

Fig. 9. Complex Mode 1 in the Nyquist map

1-norm [20]. Thus, $\operatorname{Im}\left(\psi_{\nu}\right)=-\operatorname{Re}\left(\psi_{\nu}\right)$, and the normal modes are simply estimated from the 


\subsection{Variability in test data}

complex ones by:

$$
\phi_{\nu}=\frac{\psi_{\nu}}{\sqrt{2 \mathrm{\jmath} \lambda_{\nu}}}
$$

The quality of the normal mode model, as well as the pole/residu model, is directly related to their ability to represent the structural dynamic behavior. One way to assess the quality is to examine to what extent the models are able to synthesize the measured FRFs. Quantitative tools such as the FRAC (Frequency Response Assurance Criterion) can be used to assess quantitatively this quality. However, it should be noted that the superposition requires the mass normalized normal modes which can only be obtained when the colocated FRFs are available. Let $\mu_{\nu} \in \mathbb{R}$ be the identified modal mass of the mode $\nu$ defined by

$$
\mu_{\nu}=\frac{\left(\phi_{\nu}^{i}\right)^{2}}{2 \omega_{\nu} \operatorname{Im}\left(R_{\nu}^{i i}\right)},
$$

the mass normalized normal modes $\bar{\phi}_{\nu}$ are thus obtained by

$$
\bar{\phi}_{\nu}=\frac{\phi_{\nu}}{\sqrt{\mu_{\nu}}}
$$

As observed in Figure 10, the pole/residue model as well as the mass normalized normal mode model properly match the measured FRFs. The same approach is performed for all experiments.

\subsection{Variability in test data}

Experimental modal analyses are performed in order to validate the proposed approach. The various cases are obtained by changing the added masses locations and numbers. Compared to the nominal structure, the mean mass added is around $6 \%$ whereas the standard deviation is around $0.6 \%$. The variability is then evaluated on the results of 31 experimental modal bases. 

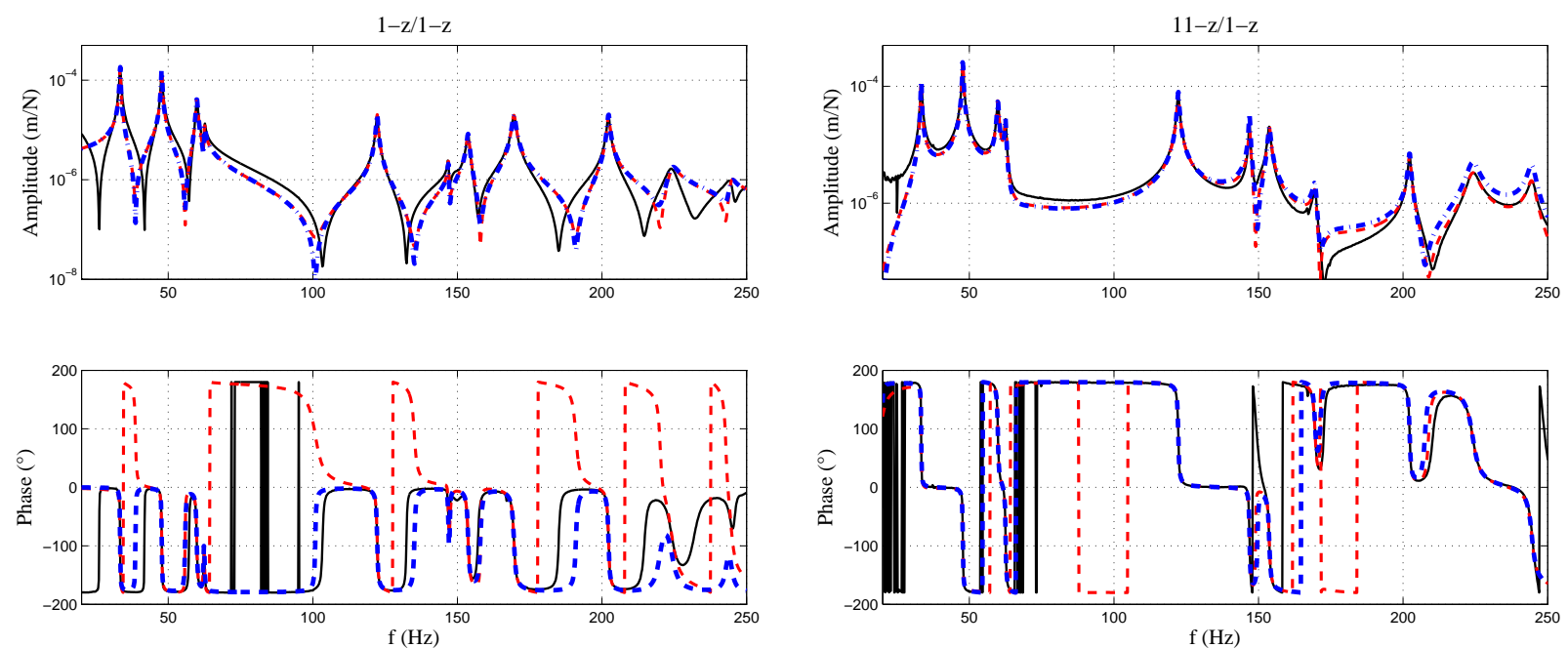

Fig. 10. Results of the modal identification for the collocated FRF and one non-collocated FRF: - measured FRFs (-), Poles/residues model (- -), Mass-normalized normal mode synthesis (- -).

\subsubsection{Variability in mode shapes, eigenfrequencies and modal damping factors}

The MAC (Modal Assurance Criterion) is a standard criterion to evaluate the correlation between two vectors [21] and is used to compare the mode shapes of the initial and modified systems. Figure 11 shows the mean (left) and standard deviation (right) of the MAC between the two systems. The number inside the MAC corresponds to the number of paired mode shapes where it is assumed that two modes are paired when their MAC is higher than 0.7 . When two modes shapes are paired, we can compare their corresponding eigenfrequencies and modal damping factors (cf. Table 1 and 2). It can clearly be observed that the variations corresponding to the perturbations of mass do not have the same impact on the different eigenproperties. In terms of frequencies, the first and fifth modes are the most affected in terms of mean, while modes 2 to 4 have almost the same mean as the nominal model. The relative standard deviation is of the same order of magnitude (7 to $11 \%$ of the mean) for the 5 modes except mode 4 whose sensitivity is much lower (about $2 \%$ of the mean). The variation of the mode shapes (Figure 11) is coherent with physical arguments, that is to say, the impact on both mean value and standard deviation is increasing with mode order. Globally, the highest variations are observed on the damping values. For all modes except mode 5, the standard deviation on the damping factor is between $18 \%$ and $31 \%$ of the mean value. For mode 5 , the standard deviation level is of the order of the mean, 


\subsection{Variability in test data}

indicating a very large variability. The mean damping factor of this mode is also highly impacted by the mass changes, while for the other modes the impact on the mean damping factor is not significant.
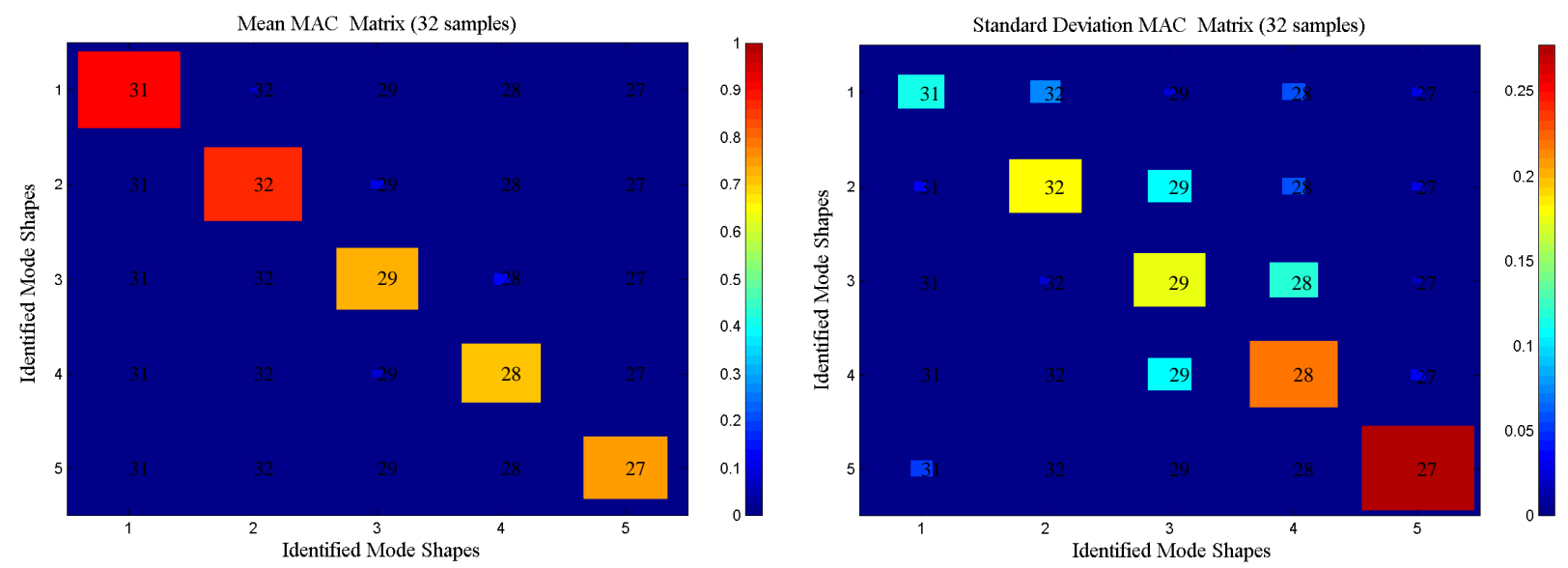

Fig. 11. Variation of the 5 first mode shapes: mean (left) and standard deviation (right).

\begin{tabular}{|c|c|c|c|c|c|c|}
\hline Mode & Initial Freq. (Hz) & Matched (over 31) & Min (Hz) & Max (Hz) & Mean Freq. (Hz) & Std. Dev. (Hz) \\
\hline 1 & 31.6 & 27 & 26.6 & 32.2 & 28.9 & 1.9 \\
2 & 45 & 29 & 36.9 & 59 & 44.3 & 5.3 \\
3 & 57.7 & 20 & 43.5 & 59.6 & 54.1 & 5.1 \\
4 & 61.9 & 17 & 58 & 62.1 & 60.5 & 1.4 \\
5 & 119.5 & 22 & 98.6 & 145.3 & 115.8 & 9.1 \\
\hline
\end{tabular}

Table 1. Variation on the eigenfrequencies

\subsubsection{Variability in the identified normal mode based FRFs}

After having detailed the variations affecting the different eigenproperties, one can observe the global variation affecting the normal mode based FRFs associated to the various configurations. Figure 12 shows the envelop FRFs for the 31 configurations (the initial system is in black) for 4 sensors. The previous conclusions are obviously still valid, in particular that the first three modes 
4.3 Construction and calibration of the info-gap model of uncertainty based on a subset of identified modal model

\begin{tabular}{|c|c|c|c|c|c|c|}
\hline Mode & Init. Damping (\%) & Matched (over 31) & Min (\%) & Max (\%) & Mean Damping (\%) & Std. Dev. (\%) \\
\hline 1 & 0.25 & 27 & 0.14 & 0.55 & 0.29 & 0.09 \\
2 & 0.27 & 29 & 0.13 & 0.47 & 0.28 & 0.08 \\
3 & 0.33 & 20 & 0.25 & 0.53 & 0.34 & 0.06 \\
4 & 0.25 & 17 & 0.20 & 0.53 & 0.30 & 0.09 \\
5 & 0.11 & 22 & 0.06 & 2.6 & 0.58 & 0.6 \\
\hline
\end{tabular}

Table 2. Variations on the modal damping factors

have similar properties in terms of frequency and damping dispersion, but also the fact that mode 5 is more dispersive both in frequency and damping. Moreover, the variability ranges of the first 3 modes are distinct from one to another.

\subsection{Construction and calibration of the info-gap model of uncertainty based on a subset of identified modal model}

The robustness function assesses the impact of eigensolution uncertainty on the performance requirement. It is therefore important that the model of uncertainty represents the correct idealization of the real uncertainty in the model. A well-known result of info-gap theory is that the more informative the info-gap model of uncertainty is, the more robust the decision making process is. This is analogous to the notion of informativeness as seen in Figure 13, where the info-gap model of uncertainty IGMU2 is more informative than the IGMU1. As a consequence, decisions based on the IGMU2 should be more robust than decision based on the IGMU1. For simplicity, it is assumed that there are no outliers in the experimental data. It is also assumed that no finite element model is available so that the best estimate is provided by the experimental modal model. Let $\mathcal{X}$ be the whole set of experimental data and $\mathcal{X}_{e}$ a subset used only to construct and calibrate the info-gap model of uncertainty. Obviously, the larger the set $\mathcal{X}_{e}$ is, the more informative the info-gap model of uncertainty will be. According to the notations introduced in section 2, $x$ is uncertain, and is in the convex set denoted $\mathcal{U}_{\boldsymbol{w}}\left(\alpha, \boldsymbol{x}^{0}\right)=\mathcal{U}\left(\alpha, \boldsymbol{x}^{0}\right)$ in this section, and expressed by equation (5). Some strategies for calibrating this uncertainty model can be found in the literature (see [22] or chap.8 in [1]). The calibration process may concern not only the parameters of the model of 

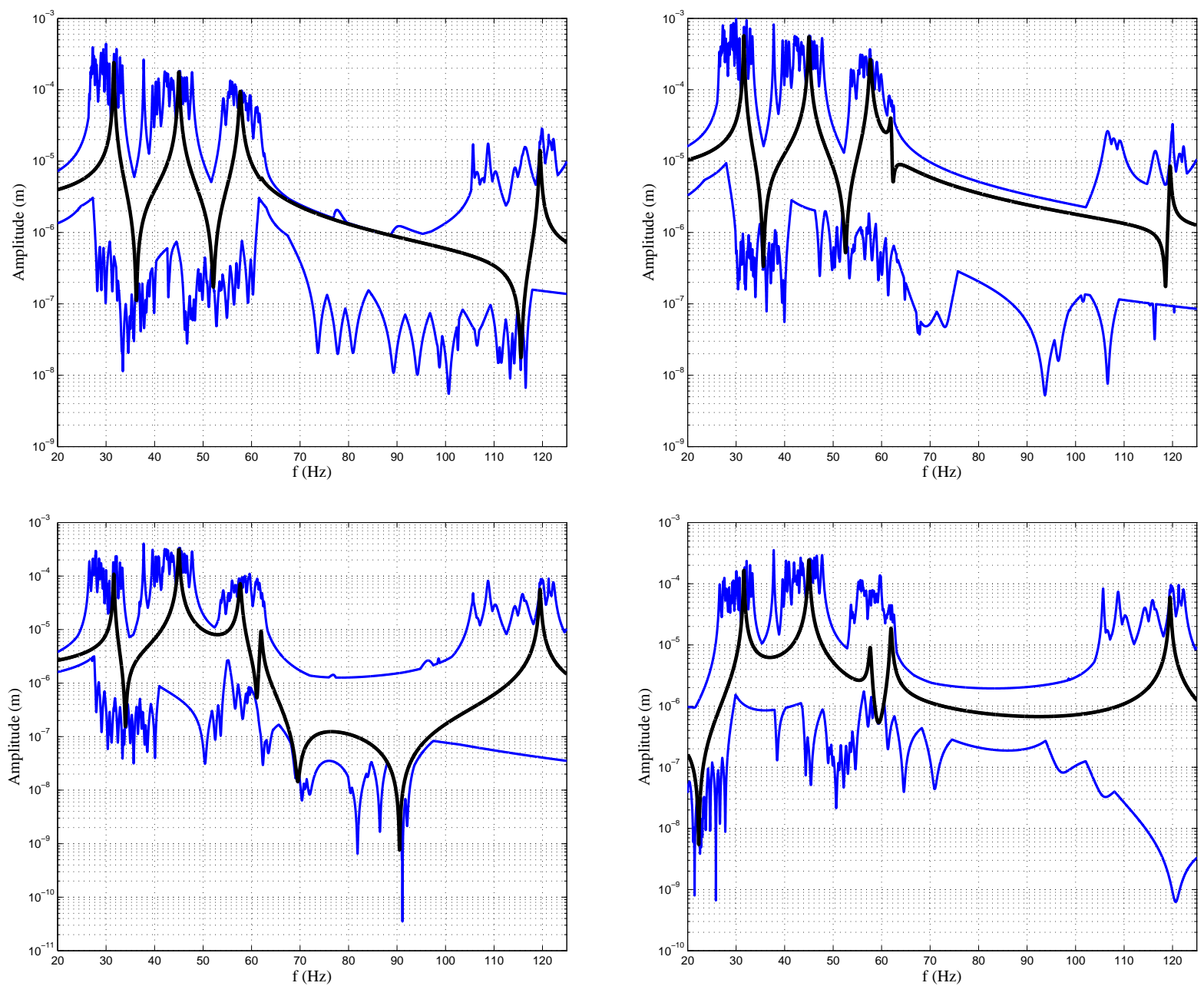

Fig. 12. Envelop mass normalized normal mode synthesis for the 31 configuration and associated to 4 sensors.

uncertainty (center or weighting coefficient) but also the structure (envelop, absolute error model, etc.). One quantitative way to assess the fidelity between experimental data and info-gap model of uncertainty is the gap-function $\Gamma\left(\mathcal{U}_{\boldsymbol{w}}\left(\alpha, \boldsymbol{x}^{0}\right), \boldsymbol{w}\right)$ defined by

$$
\Gamma\left(\mathcal{U}_{\boldsymbol{w}}\left(\alpha, \boldsymbol{x}^{0}\right), \boldsymbol{w}\right)=\min \left\{\alpha: \mathcal{X}_{e} \subseteq \mathcal{U}_{\boldsymbol{w}}\left(\alpha, \boldsymbol{x}^{0}\right)\right\}
$$

Small (resp. large) values for $\Gamma\left(\mathcal{U}_{\boldsymbol{w}}\left(\alpha, \boldsymbol{x}^{0}\right), \boldsymbol{w}\right)$ indicate a good (resp. low) agreement between the model of uncertainty $\mathcal{U}_{\boldsymbol{w}}\left(\alpha, \boldsymbol{x}^{0}\right)$ and the subset of test-data $\mathcal{X}_{e}$. Nevertheless, the main question is: 


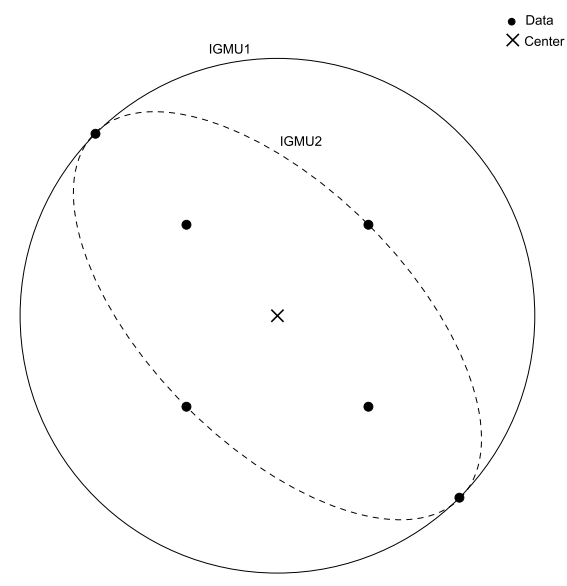

Fig. 13. Notion of informativeness (2 uncertain parameters)

does the test data provide experimental evidence against the info-gap model? Here, confidence in the ability of the structure of the model of uncertainty to idealize uncertainties is high. The problem to be addressed is the fundamental choice of the weighting vector. It is obvious that the eigenfrequencies can not be considered as more uncertain than the modal damping factors or even than the mode shapes (section 4.2). Nevertheless, the weighting vector will be considered to be constant as the uncertainty increases, though an $\alpha$-dependent or mode-dependent weighting vector can be defined. For the sake of clarity, we assume that the weighting vector which defines the uncertainty in the eigenfrequencies, denoted $\boldsymbol{w}_{\omega}$, is the unit vector. The objective here is to assess $\boldsymbol{w}_{\xi}$ and $\boldsymbol{w}_{\phi}$ based on the subset of test data. Hence, they are referred as the parameters to be calibrated in order to reduce the discrepancy between the info-gap model of uncertainty and the test data $\mathcal{X}_{e}$. The cost function used to calibrate the model of uncertainty is the empirical robustness. It is an estimate of the horizon of uncertainty within which the info-gap model captures some fraction of the measurements (ch. 8 in [1]). In our case, the empirical robustness, $\hat{\alpha}_{e}\left(\boldsymbol{w}, r_{c}\right)$, is defined by

$$
\hat{\alpha}_{e}\left(\boldsymbol{w}, r_{c}\right)=\max \left\{\Gamma\left(\mathcal{X}_{e}, \boldsymbol{w}\right): \Gamma\left(\mathcal{X}_{i}, \boldsymbol{w}\right) \leq \Gamma\left(\mathcal{X}_{e}, \boldsymbol{w}\right) \text { if } \max _{\boldsymbol{x} \in \mathcal{U}_{\boldsymbol{w}}\left(\Gamma\left(\mathcal{X}_{i}, \boldsymbol{w}\right), \boldsymbol{x}^{0}\right)} s \leq r_{c}\right\}
$$


The calibrated parameters, $\hat{\boldsymbol{w}}$, are the ones that minimize the empirical robustness:

$$
\hat{\boldsymbol{w}}=\underset{\boldsymbol{w}}{\arg \min } \hat{\alpha}_{e}\left(\boldsymbol{w}, r_{c}\right)
$$

Figure 14 shows the repartition of the modal data before calibration: the starting values for the weighting vectors are $w_{\xi}=1$ and $w_{\phi}=1$. Two test data are used to both assess and improve the model of uncertainty (depicted in red squares in Figure 14).

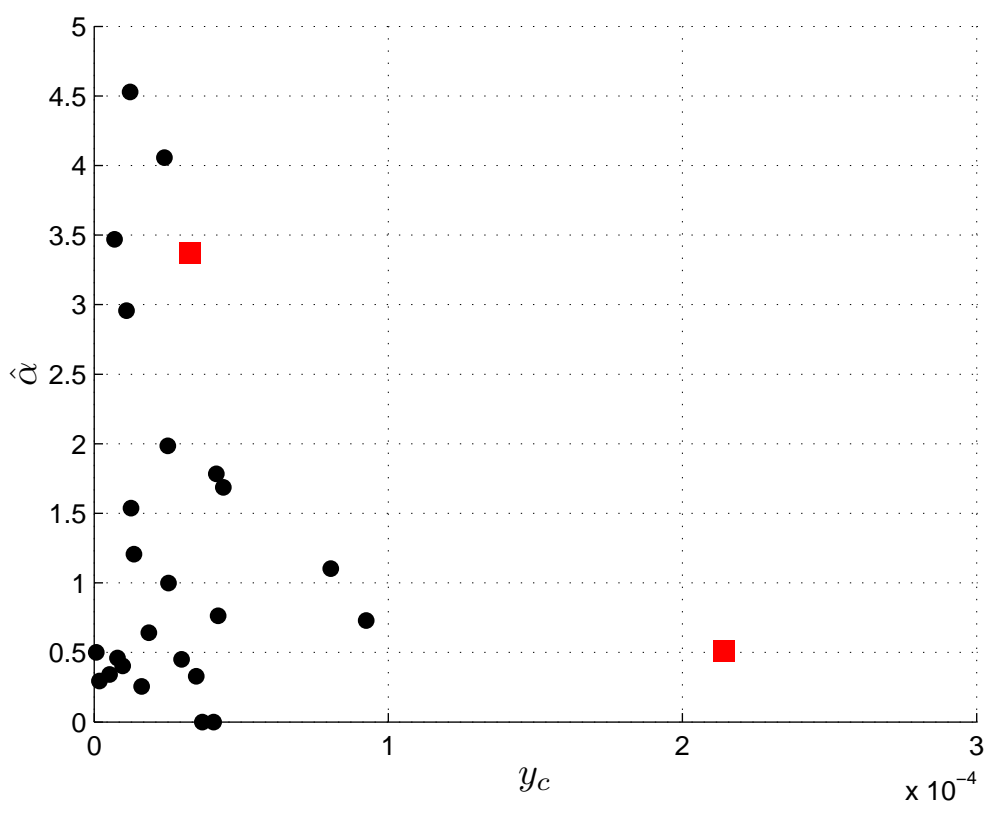

Fig. 14. Experimental data before calibration $\left(w_{\xi}=1\right.$ and $\left.w_{\phi}=1\right)$

Figure 15 shows the empirical robustness (log scale) when two test data are available. The weighting vector that best fit the model of uncertainty is the one that minimizes the empirical robustness. In this case, $w_{\xi} \geq 20.8$ and $w_{\phi} \geq 4.3$ are the minimal parameters yielding small empirical robustness values (less than 0.17 ) as indicated in Figure 15. The empirical robustness does not have a clear minimum as several pairs of values minimize the empirical robustness. In this case, any point in the zone $w_{\xi} \geq 20.8$ and $w_{\phi} \geq 4.3$ can be chosen. The selected pair $\left(w_{\xi}=25\right.$ 


\subsection{Confrontation between the model of uncertainty and real experiments}

and $\left.w_{\phi}=20\right)$ is indicated by a square in Figure 15.
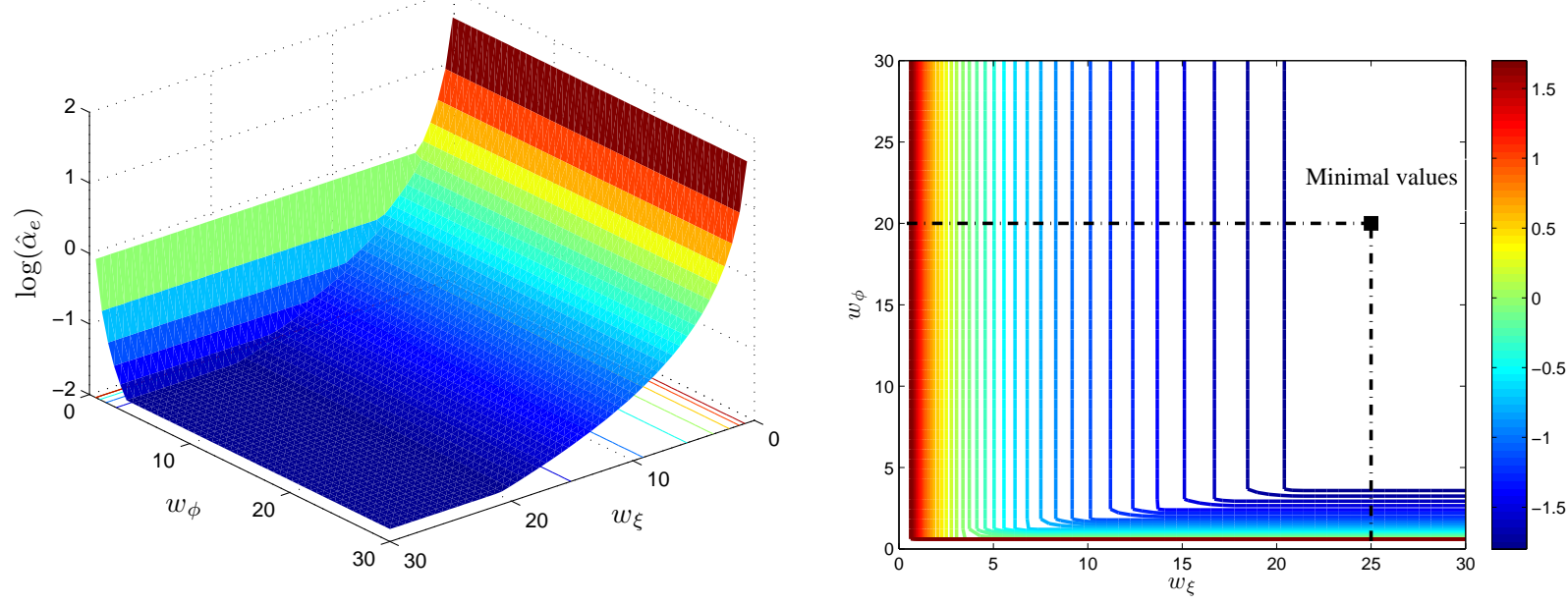

Fig. 15. Empirical Robustness $(\log )$ vs $\left(w_{\xi}, w_{\phi}\right)$ and selected values for $w_{\xi}$ and $w_{\phi}=20$.

\subsection{Confrontation between the model of uncertainty and real experiments}

The final objective of this paper is to illustrate that the calibrated model of uncertainty is consistent with real experimental data when the robustness of dynamic response levels to an uncertain modal model is evaluated. According to the approach described in section 2, a robustness analysis is performed to investigate the impact of lack of knowledge affecting the modal model on dynamic response levels. The dynamic response level is evaluated at $f=30 \mathrm{~Hz}$. The robustness curve is plotted in Figure 16. Meanwhile, test data coming from the real experiments are plotted (black circle on the Figure 16). One can observe that the robustness curve maximizes well the response levels when uncertainty is increasing. However, the model of uncertainty tends to underestimate the robustness values, though a rigorous calibration of the model of uncertainty has been performed. Several explanations for this result are possible, including for instance the topology of the uncertainty model or the fact that the weighting factors remain constant for each mode and for each value of the horizon of uncertainty. However, the approach adopted here consists in using the information available, thought additional tests could be performed in order to obtain a best model of uncertainty that represents much better the reality. 

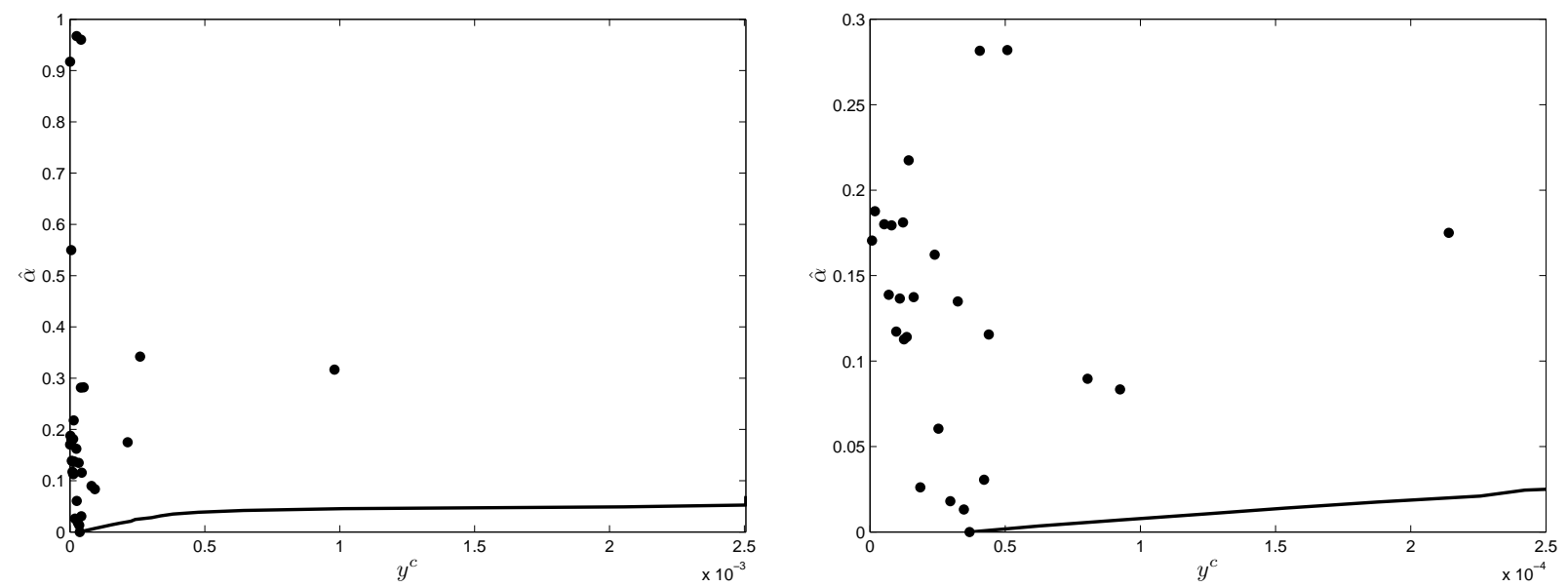

Fig. 16. Robustness curve (black curve) and test data (black circle). Zoom on the right.

\section{CONCLUSION}

This paper proposes a methodology which aims at analyzing the robustness of the dynamic response to an uncertain modal model. The objective is to provide useful bounds to the maximum response levels based only on experimental modal data. The analysis does not require probabilistic assumptions since the uncertainty is modeled by an info-gap model of uncertainty. The uncertain modal model is constructed based on information provided by the initial modal model. An info-gap robustness analysis is first illustrated on a simple finite element model. A series of experimental modal analyses are then performed on a structure composed of two plates clamped together on one side. The uncertainty is introduced in the system by adding lumped masses at random locations. A first info-gap robustness analysis indicates that the a priori model of uncertainty does not match very well with experiments and over-estimates the robustness function. The info-gap model of uncertainty is then calibrated using a subset of test data with the purpose of improving the fidelity to the subset data. Finally, it is shown that, after the calibration procedure, the info-gap model of uncertainty is consistent with real test data and provides a more realistic robustness curve.

Future work will focus on improving the model of uncertainty by performing additional tests on different systems. 


\section{REFERENCES}

[1] Ben-Haim, Y., 2006. Information-Gap Theory: Decisions Under Severe Uncertainty. 2nd edition, Academic Press, London.

[2] Knight, F., 1921. Risk, Uncertainty and Profit. Houghton Mifflin Co.

[3] Roy, B., 2010. "Robustness in operational research and decision aiding: A multi-faceted issue". European Journal of Operational Research, 200(3), pp. 629 - 638.

[4] Oberkampf, W. L., DeLand, S. M., Rutherford, B. M., Diegert, K. V., and Alvin, K. F., 2002. "Error and uncertainty in modeling and simulation". Reliability Engineering \& System Safety, 75(3), pp. $333-357$.

[5] Klir, G., 2004. "Generalized information theory: aims, results and open problems". Reliability Engineering and System Safety, 85, pp. 21-38.

[6] Kiureghian, A. D., and Ditlevsen, O., 2009. "Aleatory or epistemic? does it matter?". Structural Safety, 31(2), pp. $105-112$.

[7] Ghanem, R., and Spanos, P., 1991. Stochastic Finite Element: A Spectral Approach. Springer-Verlag.

[8] Soize, C., 2000. "A nonparametric model of random uncertainties for reduced matrix models in structural dynamics". Probabilistic Engineering Mechanics, 15(3), pp. 277 - 294.

[9] Schuëller, G., 2007. "On the treatment of uncertainties in structural mechanics and analysis". Computers \& Structures, 85, pp. $235-243$.

[10] Adhikari, S., 2008. "Wishart random matrices in probabilistic structural mechanics". Journal of Engineering Mecahnics, 134(12), pp. 1029-1044.

[11] Dessombz, O., Thouverez, F., Laîné, J.-P., and Jézéquel, L., 2001. "Analysis of mechanical systems using interval computations applied to finite element methods". Journal of Sound and Vibration, 239(5), pp. 949 - 968.

[12] Moens, D., and Hanss, M., 2011. "Non-probabilistic finite element analysis for parametric uncertainty treatment in applied mechanics: Recent advances". Finite Elements in Analysis and Design, 47(1), pp. $4-16$.

[13] Dempster, A., 1968. "Upper and lower probabilities generated by a random closed interval". 
REFERENCES

The Annals of Mathematical Statistics, 39(3), pp. 957-966.

[14] Shafer, G., 1976. A mathematical theory of evidence, Vol. 1. Princeton university press Princeton.

[15] Adhikari, S., and Sarkar, A., 2009. "Uncertainty in structural dynamics: Experimental validation of a wishart random matrix model". Journal of Sound and Vibration, 323, pp. $802-$ 825.

[16] Adhikari, S., Friswell, M., Lonkar, K., and Sarkar, A., 2009. "Experimental case studies for uncertainty quantification in structural dynamics". Probabilistic Engineering Mechanics, 24(4), pp. 473-492.

[17] Friswell, M., Coote, J., Terrell, M., Adhikari, S., Fonseca, J., and Lieven, N., 2005. "Experimental data for uncertainty quantification". In Proceedings of the IMAC XXI.

[18] Pichler, L., Pradlwarter, H. J., and Schuëller, G. I., 2008. A mode-based meta-model for the frequency response functions of uncertain structural systems.

[19] Guillaume, P., Verboven, P., Vanlanduit, S., der Auweraer, H. V., and Peeters, B., 2003. "A poly-reference implementation of the least-squares complex frequency-domain estimator". In Proceedings of the IMAC XXI.

[20] Balms, E., 1997. "New results on the identification of normal modes from experimental complex modes". Mechanical Systems and Signal Processing, 11(2), pp. 229-243.

[21] Allemang, R., 2003. "The modal assurance criterion: twenty years of use and abuse.". Journal of Sound and Vibration, 37(8), pp. 14-23.

[22] Ben-Haim, Y., 2004. "Uncertainty, probability and information-gaps". Reliability Engineering \& System Safety, 85, pp. $249-266$.

[23] Göhler, M., Eifler, S., and Howard, T., 2016. "Robustness metrics: Consolidating the multiple approaches to quantify robustness". ASME. J. Mech. Des., 138(11): 111407, Nov.

[24] Zhang, M. Q., Beer, M., Koh, C. G., and Jensen, H. A., 2016. "Nuanced robustness analysis with limited information". ASCE-ASME Journal of Risk and Uncertainty in Engineering Systems, Part A: Civil Engineering, 2(3), p. B4015001.

[25] Atamturktur, S., Liu, Z., Cogan, S., and Juang, H., 2015. "Calibration of imprecise and inac- 
curate numerical models considering fidelity and robustness: a multi-objective optimizationbased approach". Structural and Multidisciplinary Optimization, 51(3), pp. 659-671.

[26] Stevens, G., Van Buren, K., Wheeler, E., and Atamturktur, S., 2015. "Evaluating the fidelity and robustness of calibrated numerical model predictions". Engineering Computations.

[27] Jaboviste, K., Sadoulet-Reboul, E., Peyret, N., Arnould, C., Collard, E., and Chevallier, G., 2019. "On the compromise between performance and robustness for viscoelastic damped structures". Mechanical Systems and Signal Processing, 119, pp. 65-80.

[28] Hot, A., Weisser, T., and Cogan, S., 2017. "An info-gap application to robust design of a prestressed space structure under epistemic uncertainties". Mechanical Systems and Signal Processing, 91, pp. 1-9. 